\section{Soviet-style cell motility}

\author{
Dennis Bray
}

Cytoskeleton. By Alexander D. Bershadsky and Juri M. Vasiliev. Plenum: 1988. Pp.298. \$47.40, £27.90.

SCIENCE monographs have become such a stylized feature of Anglo-American culture that it seems strange to find one of the genre emerging from the Soviet Union. Indeed, this glossy volume, written in impeccable English and full of up-to-date references and micrographs, is almost uncannily good - as though the two Muscovites had taken centre stage in a Country and Western show with a twangy rendition of "Mommas, don't let your babies grow up to be cowboys". Where did they learn to give such a polished performance? How did they - with all the difficulties that are said to exist in the Soviet Union in obtaining Western periodicals and travelling to conferences - become so well informed? Whatever the answer, this volume sends a clear signal that at least one active and critical school of cell biology has survived the Siberian winter. May it flourish now that Spring is on its way!

This is the best book on the cytoskeleton yet written. To be sure, one expects improvements as the field matures; as the flurry of newly discovered cytoskeletal proteins begins to settle, we expect to discern the contours of a larger structure beneath. But the authors have not missed any opportunities in the integration of their material. A good half of the book is devoted to the organization of cytoskeletal elements - links between different sets of filaments are examined as well as their association with the plasma membrane and the cell nucleus. One chapter deals with the interplay of cytoskeletal elements during cell locomotion, another with cell division. The final chapter on the neoplastic transformation of fibroblasts, a research interest of the authors, concerns the fascinating links between oncogenes and the cytoskeleton.

Most of the important new developments have made it into the book. Dynamic instability and kinesin, integrin and talin, intermediate filament sequences, control over tubulin mRNA stability - all are mentioned, although some very briefly. The text is systematically organized, with each filament system being introduced in turn and each cytoskeletal protein or mechanism or drug receiving its allocation of space. Areas of uncertainty or argument (of which, in this field, there are a large number) are dissected with scrupulous impartiality: "Here are the possible mechanisms, A, B, and
C", the authors seem to say, "And here is the evidence for each of them, in turn". It is a scientific style for a scientific book; appropriate, I suppose, but the result is a little dry and joyless. This is probably not a volume to capture a young person's imagination.

But then the book was not written as a student text on cell movements. It is, explicitly, a monograph in a series on cellular organelles, the organelle in question being the cytoskeleton. As such, it per-

\section{Portrait of a cell}

\section{John Sommerville}

Paramecium. Edited by H.-D. Görtz. Springer-Verlag:1988. Pp.444. DM248, 183.

TO DEVOTE a whole volume to a single eukaryotic cell may seem an extravagance. But not in this instance, for Paramecium is one of our favourite organisms.

Superficially familiar to students as a busy little forager spiralling through pondwater, Paramecium has much to offer the modern research worker. Indeed, the ciliated protists as a group are among the most fascinating of all cells, having evolved to a level of complexity rarely seen elsewhere. Complexity is evident not only in their intricate structural features but also in their genetic mechanisms, metabolic processes and locomotor activities. Thus many of the features of higher organisms - differentiation, responsive behaviour, a digestive system - are seen here in a single cell, and the endeavours to understand them, as manifested in Paramecium, are reviewed in this volume.

Although Paramecium is accessible to a wide variety of experimental approaches, progress has been limited by some technical problems. One is the difficulty associated with cell culture in axenic media (Paramecium eats bacteria in nature); another is the dearth of suitable (micronuclear) mutations for genetic analysis. Happily, progress in both of these areas is reported in the book.

These and other problems and prospects with using Paramecium as an experimental organism are identified by John Preer, whose foreword provides a useful overview of various studies described in the text. Also, an early chapter by Richard Allen gives the background to the ultrastructural aspects of the systems which are tackled in more detail later on. Each system (ranging through taxonomy, mating types, cell cycle, nuclei, immobilization antigens, mitochondria, ion channels, cilia, chemokinesis, lysosomes, trichocysts, cytoskeleton, endosymbionts, ecology) is the subject of a separate contribution written by appropriate specialists. Although the 24 chapters vary in length and detail of content, each is clearly writ- forms its function to an exemplary degree, with many useful tables and a wealth of original, somewhat idiosyncratic, line drawings. Complete, accurate and insightful, it will be a valuable resource for research workers and a useful supplementary text for courses in cell biology. I hope it does well.

Dennis Bray is in the Medical Research Council Cell Biophysics Unit, King's College London (KQC), 26-29 Drury Lane, London WC2B SRL, UK.

ten and amply referenced. Related topics are grouped together, and format is consistent throughout, resulting overall in a coherent, well-integrated volume.

Yet several aspects of Paramecium remain enigmatic or involve biological principles which we are only beginning to understand. These aspects transcend the boundaries of individual chapters. For instance, nuclear dimorphism, whereby intact copies of the genome are preserved in micronuclei, and amplified, fragmented, deleted and rearranged copies are expressed in macronuclei, provides a model not only for germ line versus soma effects (Chapter 8) but also for chromatin activation (Chapter 10) and the nature of ageing (Chapter 9). Differentiation and inheritance of cortical structures (Chapters 16,20 and 21) relate to the construction and reproduction of complex supramolecular assemblies by mechanisms not yet understood. The relationship between excitable membranes (Chapter 13), ionchannels (Chapter 15), motility (Chapter 14) and locomotor behaviour (Chapter 18) become clearer through the analysis of behavioural mutants (Chapters 15 and 17). Patterns of cytoplasmic inheritance, through mitochondria (Chapter 12) and bacterial endosymbionts (Chapters 22-24), or through less well defined 'cytoplasmic states' which can control macronuclear gene expression (foreward and Chapters 4 and 11), involve interactions between different genomes.

Paramecium presents us with a rich canvas. Although this is not the only book available on the organism, or on ciliates in general, it does contain a comprehensive coverage of recent advances and illustrates that the details on that canvas are only just beginning to be filled in.

John Sommerville is in the Department of Biology and Preclinical Medicine, University of St Andrews, St Andrews, Fife KY16 9TS, UK.

\section{New in paperback}

- Forever Undecided: A Puzzle Guide to Gödel by R. Smullyan. Publisher is Oxford University Press, price is $£ 5.95$. For review see Nature 328, 212 (1987).

- Speakable and Unspeakable in Quantum Mechanics by J.S. Bell. Publisher is Cambridge University Press, price is $£ 8.95, \$ 14.95$. For review see Nature 331, 667 (1988).

- Was Einstein Right? by C.M. Will. Publisher is Oxford University Press, price is $£ 5.95$. For review see Nature 324, 191 (1986). 